\title{
Norois
}

Environnement, aménagement, société

$210 \mid 2009 / 1$

L'agriculture et les filières agro-alimentaires en France et en Argentine

\section{La maîtrise de l'eau pour l'agriculture en Limousin. Un exemple de gestion de la ressource en moyenne montagne océanique}

Controlling water for the agriculture in the Limousin, France. An example of water management in oceanic medium mountains

El dominio del agua para la agricultura en la región Limousín (Francia). Un ejemplo de gestión del agua en la media montaña oceánica

\section{Françoise Ardillier-Carras}

\section{(2) OpenEdition}

Journals

Édition électronique

URL : https://journals.openedition.org/norois/2746

DOI : $10.4000 /$ norois. 2746

ISBN : 978-2-7535-1558-1

ISSN : 1760-8546

Éditeur

Presses universitaires de Rennes

Édition imprimée

Date de publication : 1 mars 2009

Pagination : 27-42

ISBN : 978-2-7535-0844-6

ISSN : 0029-182X

Référence électronique

Françoise Ardillier-Carras, « La maîtrise de l'eau pour l'agriculture en Limousin. Un exemple de gestion de la ressource en moyenne montagne océanique », Norois [En ligne], 210 | 2009/1, mis en ligne le 01 mars 2011, consulté le 14 janvier 2022. URL : http://journals.openedition.org/norois/2746 ; DOI : https://doi.org/10.4000/norois.2746

Ce document a été généré automatiquement le 14 janvier 2022.

(ㄷ) Tous droits réservés 


\title{
La maîtrise de l'eau pour l'agriculture en Limousin. Un exemple de gestion de la ressource en moyenne montagne océanique
}

\author{
Controlling water for the agriculture in the Limousin, France. An example of \\ water management in oceanic medium mountains \\ El dominio del agua para la agricultura en la región Limousín (Francia). Un \\ ejemplo de gestión del agua en la media montaña oceánica
}

Françoise Ardillier-Carras

\section{NOTE DE L'ÉDITEUR}

Cet article a été reçu le 13 juin 2008 et définitivement accepté le 21 février 2009.

1 Fait de civilisation, la maîtrise de l'eau pour la mise en valeur agricole témoigne des liens ancestraux entre l'Homme et sa terre, sous un double sceau : soumission et exploitation, préfigurant des relations complexes et nuancées. En France, les superficies irriguées sont estimées à $10 \%$ de la SAU cultivée en 2003. Les surfaces irrigables ont été multipliées par 4,5 de 1961 à 1996. Cette évolution ne concerne pas seulement les régions sèches, de type méditerranéen. Alors que l'eau est relativement abondante dans les régions occidentales, bénéficiant de précipitations d'Ouest, les caprices du climat océanique peuvent générer des contraintes, par manque ou par excès. C'est le cas du Limousin. Dans ce contexte paradoxal, où l'abondance n'exclut pas la pénurie, la maîtrise de l'eau pour les besoins agricoles spécifiquement liés à la production d'herbe relève de principes de gestion raisonnée, en grande partie hérités. En Limousin, l'eau fait figure de potentiel économique mais il est toujours apparu que sa valorisation reste faible en raison d'une utilisation extensive, archaïque et 
incohérente (Balabanian et Bouet, 1989). Cette distorsion entre abondance et faible valorisation soutient cette réflexion où, parallèlement aux principes de maîtrise de l'eau, il convient de mettre l'accent sur les conditions présidant à la gestion de l'eau pour l'agriculture aujourd'hui.

2 Située sur la terminaison nord occidentale du Massif central, la région du Limousin est représentative de situations contrastées qui ne la mettent pas à l'abri de pénuries ou de surcroît d'humidité. Cette singularité influence, depuis toujours, l'activité agricole de cette région, "pays de l'arbre et de l'eau ${ }^{1}$ ", à vocation herbagère. Dans ce contexte, gérer la petite hydraulique agricole en vue d'une utilisation raisonnée de la ressource en eau a toujours été une préoccupation essentielle pour les paysans du Limousin. Aujourd'hui, le développement agricole reste largement conditionné par les pratiques de gestion de l'eau, mais est aussi de plus en plus commandé par les impératifs économiques des politiques agricoles - en particulier de l'Union européenne - et la montée des préoccupations environnementales. Les réglementations en vigueur contribuent largement à modifier les pratiques agricoles pour protéger la ressource et les milieux aquatiques.

\section{Quand l'eau subie devient l'eau conquise. Contraintes, risques et héritages}

\section{Un milieu, des contraintes : l'espace agricole de moyenne montagne}

3 Le Limousin appartient à cette moyenne montagne qualifiée de " fragile ", bastion agricole touché par la dépopulation et la récession économique avec recul généralisé de l'agriculture. Les caractères physiques conditionnent en partie la mise en valeur agricole, mais les contraintes du milieu n'expliquent pas tout. La situation de déprise relève aussi d'héritages socio-économiques, de mutations dans les pratiques agricoles et de l'influence de la conjoncture. Dans cette configuration, la maitrise de l'eau reste un important facteur de développement agricole (fig. 1). Le Limousin occupe une position d'amont, partie du château d'eau du Massif central. A partir des hautes terres granitiques du plateau de Millevaches (« Montagne limousine », à l'est, culminant à 978 $\mathrm{m})$, la topographie du Limousin s'abaisse vers l'ouest en une succession de plateaux étagés incisés par les vallées. Ces traits de caractère ne préfigurent en rien l'adéquation entre capacités d'alimentation en eau - y compris via le réseau hydrographique - et besoins agricoles. "Le Limousin est, en quelque sorte, victime d'être, dans son ensemble, le premier massif montagneux que rencontrent sur leur chemin les précipitations venant de l'ouest et du nord-ouest " (Balabanian et Bouet, 1989). Cette situation, qui n'est ni isolée, ni limitée à cette région, prend une importance particulière : qui pourrait se soucier de maîtriser l'eau dans une telle configuration d'abondance relative? 
Figure 1. Le Limousin : localisation et relief The Limousin region in France

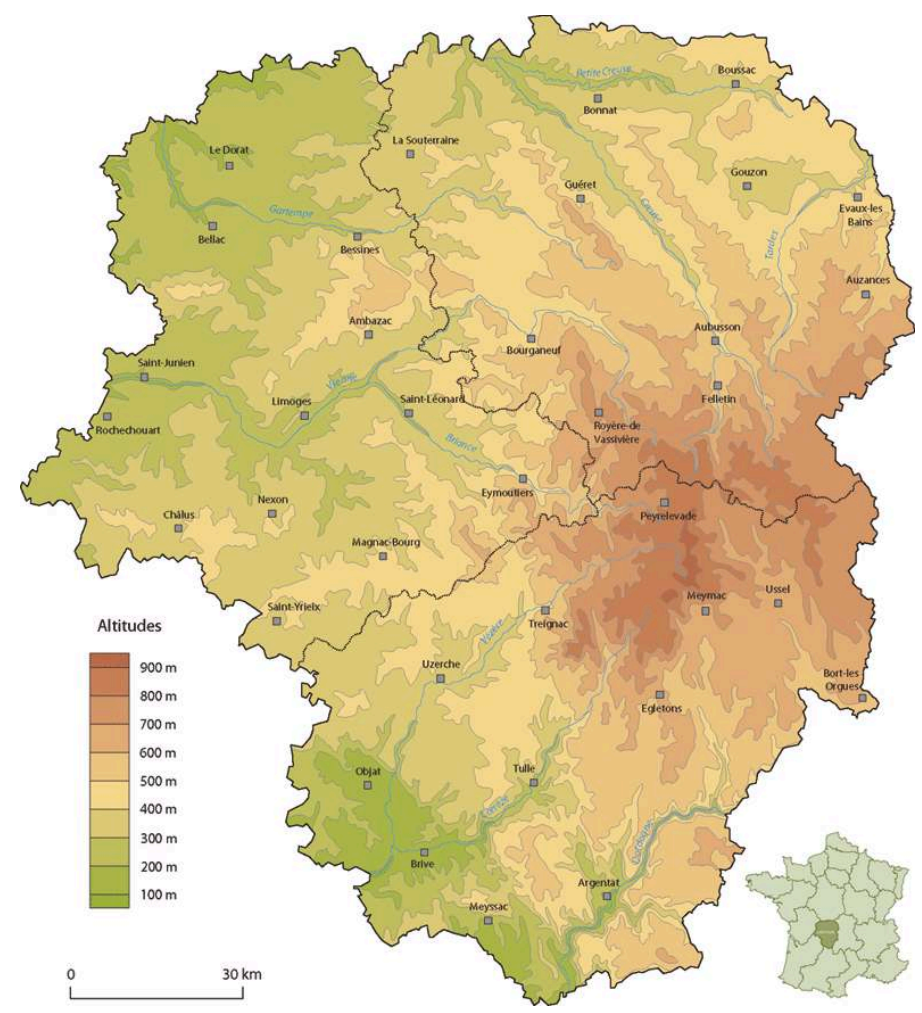

Les caractéristiques topographiques et climatiques de la région en font un véritable " château d'eau ", donnant naissance à un réseau hydrographique dense - plus de 11000 $\mathrm{km}$ de cours d'eau : Vienne, Gartempe, Vézère. Le climat limousin, de type océanique dégradé, est caractérisé par des pluies abondantes, dont les excès ne compensent jamais les manques. "Il s'agit d'un climat qui engendre tour à tour et à la fois l'insatisfaction, l'exaspération et finalement la sagesse » (ibidem). Avec une pluviométrie annuelle allant de 1600 à $1400 \mathrm{~mm} / \mathrm{an}$, à l'est, sur les hautes terres de la Montagne limousine (près de $1000 \mathrm{~m}$ d'altitude), à 850 à $900 \mathrm{~mm}$ à l'ouest et au nordouest, sur les bordures poitevine et berrichonne, en relation avec leur topographie de plateaux étagés (Bellac est à $230 \mathrm{~m}$ ). Au total, il pleut beaucoup en Limousin, $1000 \mathrm{~mm} /$ an en moyenne. Dans cette portion de massif ancien granitique, les bassins versant réagissent fortement à la pluviométrie par un ruissellement important. La prédominance des écoulements de surface est un trait majeur de la configuration hydrologique du milieu, alors qu'en période de sécheresse, les débits ne sont pas soutenus par des réserves souterraines, d'où les fortes variations de la réserve en eau qui interfèrent fondamentalement dans la disponibilité de la ressource selon les épisodes pluviométriques. Sur cette bordure du Massif central, les sols, imperméables, développés sur roche mère granitique ou métamorphique, passent, progressivement, sur les marges nord et ouest, à des éléments sédimentaires, via les formations sidérolithiques au contact entre Massif central et Poitou ou Berry au nord, ou encore vers le bassin de Brive, au sud-ouest.

5 Les prairies humides, ou mouillères, localisées dans les fonds de vallées peuvent être assainies grâce à l'élimination des excès d'eau par le drainage. Mais ce n'est pas le seul facteur : traditionnellement entretenues pour le pâturage bovin, elles subissent surtout les effets de la déprise agricole. Excès et manques d'eau, caractères essentiels de la 
relation entre eau et agriculture dans cette région de moyenne montagne océanique, sont en étroite relation avec la répartition des précipitations, l'irrégularité du volume précipité, la durée variable des séquences humides.

Photo 1 : Mouillère en tête de bassin, vallée de la Gartempe dans la Creuse. Pâturages en partie drainés (en rive gauche) (Cliché $@$ C F. Ardillier)

Wet plot in the headwater catchment, valley of the Gartempe river. Meadows partly drained

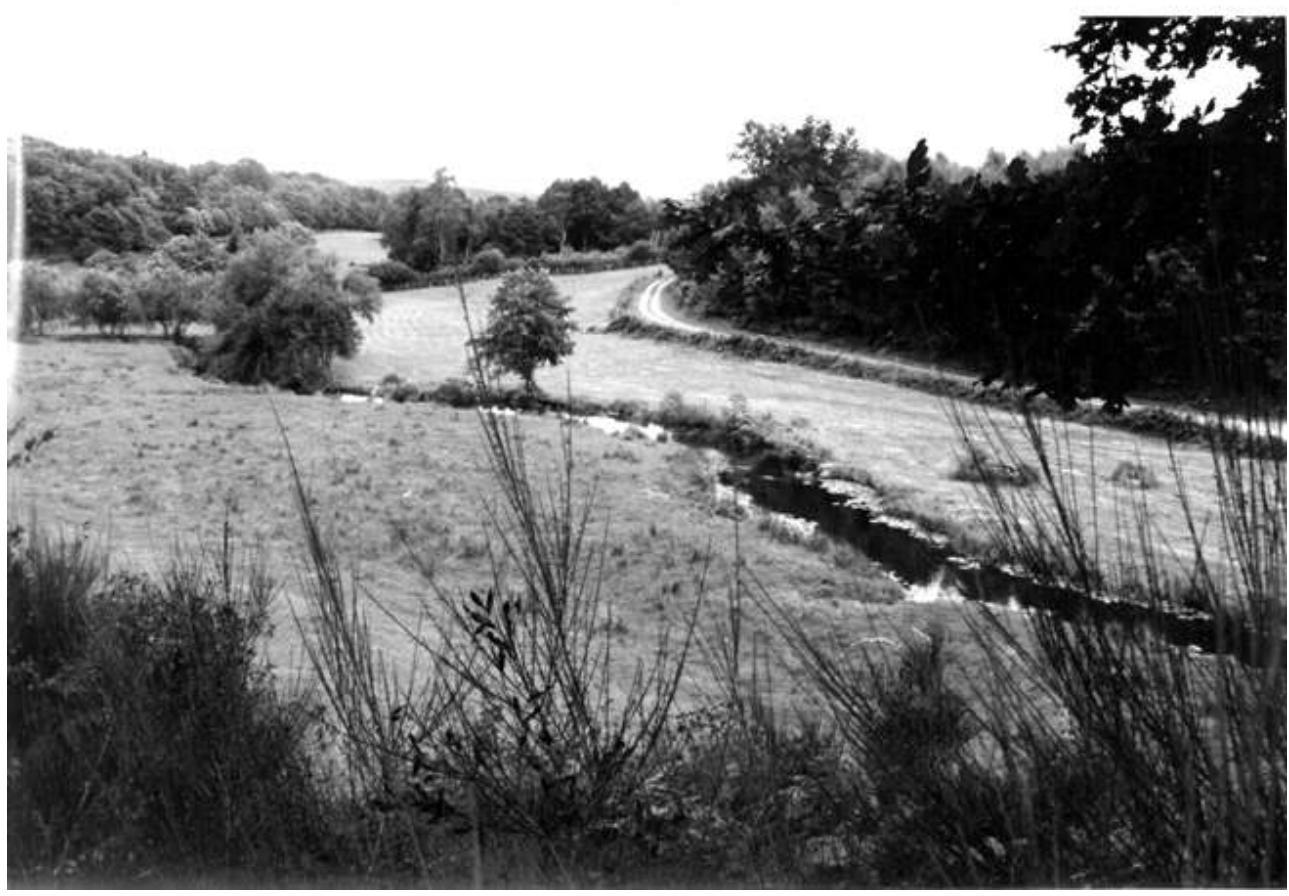

La mobilisation de la ressource en eau correspond à une prise de conscience de l'exploitant face aux composantes du milieu. L'agriculteur qui a opté pour le drainage se trouve introduit dans un système nouveau où l'eau devient maîtrisable. L'irrigation vient ensuite, presque logiquement, pour compléter le processus. Cette évolution ne procède pas de la seule technique, mais d'une démarche lente et profonde qui va chercher ses racines dans les mentalités marquées par un héritage. C'est ainsi que l'eau « subie » devient « eau conquise » et facteur de développement agricole.

\section{L'hydraulique agricole : un héritage du Moyen Âge}

7 En agriculture, la relation à l'eau était autrefois marquée par la soumission aux contraintes. L'eau était " subie " et son utilisation, étroitement tributaire des fluctuations climatiques et hydrologiques. Irriguer en Limousin, alors que l'excès d'eau dans les sols est un problème majeur, ne semble pas être, à première vue, une opération indispensable; or, non seulement cette pratique existe depuis des siècles, mais elle progresse, avec des objectifs nouveaux et de nouvelles méthodes.

ès le Moyen Âge, on irriguait les prairies naturelles sur les versants. Les ordres religieux installés dans la région, comme les moines cisterciens, au XII ${ }^{\mathrm{e}}$ siècle, avaient réussi à coloniser certains espaces répulsifs grâce à des techniques hydrauliques ingénieuses pour, à la fois, irriguer les sols secs et mettre en valeur les fonds naturellement trop humides. De la même manière, les seigneurs, propriétaires fonciers, 
associèrent également, suivant ces principes, maitrise de l'eau pour l'agriculture, exploitation de l'énergie hydraulique et production de poissons.

Les paysages du XIX ${ }^{e}$ siècle (Perpillou, 1950) où de vastes étendues de landes, d'ajoncs et de bruyère couvrent la majeure partie de l'espace agricole, traduisent une carence d'innovation. Les terres les plus ingrates avaient vocation de terrains de parcours pour les troupeaux de moutons, une des bases de l'activité d'élevage dans l'ouest de la région, y compris sur ses marges poitevines. Les systèmes de production étaient basés sur les nécessités de l'alimentation locale. Seules les prairies de fauche occupant les versants sont valorisées, notamment pour assurer une pousse d'herbe d'avant saison, par le procédé d'irrigation thermique des prairies. L'irrigation des prés de fauche montre l'intérêt porté à ces parcelles à forte valeur foncière ${ }^{2}$, supports de production herbagère ${ }^{3}$.

Depuis longtemps, les paysans limousins savent limiter les risques inhérents à ces périodes sèches grâce à un système d'irrigation des prés constitué de petites rigoles, appelées localement " levades ", fossés creusés sur le versant dans le sens des courbes de niveau. Alimentées par des pêcheries, petits bassins édifiés en haut de la pente où l'eau de source est collectée et stockée, elles courent avec une faible pente. C'est par débordement et gravité que l'eau de ces rigoles arrose la zone du pré située immédiatement à l'aval de la « levade ». Cette pratique ancienne, encore très utilisée au XIX ${ }^{e}$ siècle - et très occasionnelle aujourd'hui - avait frappé Arthur Young (xVIII ${ }^{e}$ siècle) qui estimait qu'elle était réalisée avec grand soin, ce qui pour le voyageur anglais « marquait combien on se rendait compte de l'importance de cette amélioration ». Les " levades », réalisées surtout vers la fin de l'hiver, remplissent principalement une fonction thermique : apporter des degrés pour activer la pousse de l'herbe dès la fin de la période froide. L'eau ainsi répandue, toujours moins froide que le sol, protégeait le manteau herbeux du gel et avançait la pousse de l'herbe au début du printemps de trois à quatre semaines, tout en protégeant l'herbe contre d'éventuels gels nocturnes. 
Photo 2 : Une " pêcherie ", réserve d'eau en haut de pente servant autrefois à alimenter les levades pour l'irrigation thermique en contrebas (ici dans les monts de Blond [515 m]) (cl. F. Ardillier-Carras) A "pêcherie", water supply in the high part of the slopes, formerly to fill the "levades" for a thermic irrigation below (here in the Mounts of Blond [515m])

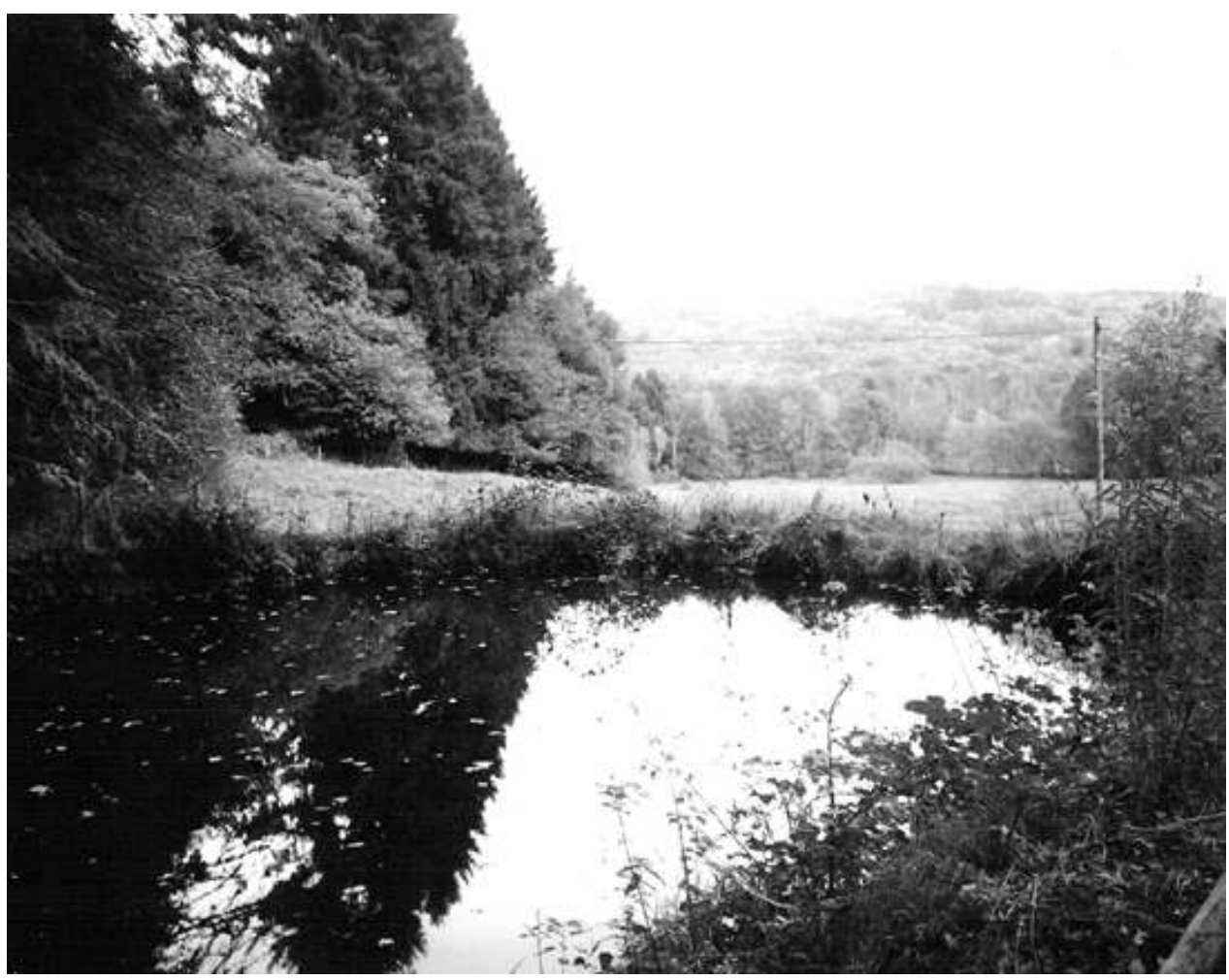

Cette technique ${ }^{4}$, pratiquée à la fin de l'hiver et au printemps, favorisait la repousse de l'herbe destinée à être fauchée, permettant ainsi parfois 2 ou, exceptionnellement, 3 fauches supplémentaires et d'avant-saison. Par ailleurs, de façon seconde, l'eau ainsi répandue en fin de printemps ou en été - stockée dans les pêcheries - permettait de lutter contre les éventuelles sécheresses.

12 En fait, les préoccupations d'aujourd'hui en Limousin sont les mêmes qu'hier, quoique d'échelle différente : stocker l'eau pour parer aux sécheresses (étangs), valoriser les fonds humides (étangs), éliminer l'excès d'eau du sol (drainage).

\section{Les pratiques de gestion de l'eau au service de la production agricole aujourd'hui en Limousin}

Les objectifs contemporains de production et de mise en valeur agricole confèrent à l'utilisation de l'eau pour l'agriculture bien des caractères nouveaux, commandés, pour une large part, par l'évolution des techniques et la recherche de la compétitivité.

Certaines productions sont valorisées par le marché et privilégiées par les programmes européens, parmi lesquelles les cultures à forte valeur ajoutée, telles que fruits et légumes, vignoble. Or, les régions de moyenne montagne, avec leur tradition herbagère d'élevage bovin ou ovin, deviennent plus vulnérables, avec une moindre capacité à se développer. Dans le bassin allaitant du Massif central auquel appartient le Limousin, l'accent est mis sur les techniques d'élevage bovin viande et la production végétale pour l'alimentation du bétail. C'est là, précisément, alors que la rentabilité des 
productions animales reste la moins élevée, que l'hydraulique agricole se présente encore davantage comme un outil de dynamique productive.

Reste l'impérieuse nécessité de maîtriser les volumes et la qualité de l'eau, y compris dans cette partie de château d'eau du Massif central. Quant au bilan hydrologique, dans les conditions réelles du milieu, l'échelle pertinente n'est pas l'échelle nationale mais bien l'échelle du bassin-versant.

Avec l'exemple du bassin-versant de la Vienne, dont le périmètre englobe largement une partie du Limousin ainsi que de ses bordures poitevines, on peut mettre en évidence l'importance spatiale des surfaces irriguées et drainées.

Maitriser la variabilité de l'approvisionnement en eau, dans cette configuration de relative abondance, repose sur des principes de gestion qui s'imposent impérativement pour développer l'élevage à l'herbe. Une région à forte vocation herbagère, comme le Limousin, reste vulnérable aux impacts de variabilité climatique. La réussite de la production animale repose sur la capacité à réagir aux fluctuations pluviométriques, voire, à les anticiper. Sachant que $86 \%$ de la SAU est constituée de prairies (artificielles et STH) et que $61 \%$ du revenu agricole régional repose sur des productions animales (576 M€ dont $376 \mathrm{M} €$ en gros bovins viande et $201 \mathrm{M} €$ en fourrages), on prend la mesure de ce que signifie la valorisation des productions qui, dans ce contexte de moyenne montagne, ne peuvent donner leur pleine mesure sans une rigoureuse gestion des supports herbagers et fourragers par la maîtrise de l'eau.

\section{Stocker pour parer aux manques d'eau : I'irrigation}

Les productions animales sont plus sensibles à la sécheresse que les productions végétales stricto sensu pour deux raisons :

- à degré de sécheresse égal, la production du blé baisse moins que celle des fourrages ;

20 - la consommation des animaux étant peu flexible sur une longue période, l'autoprotection est indispensable pour l'éleveur s'il ne veut pas « décapitaliser » en réduisant son cheptel.

21 C'est ainsi que les élevages bovins, laitiers ou à viande, et d'ovins, à l'alimentation dépendant presque exclusivement de la production herbagère, sont les plus vulnérables à la sécheresse car ils dépendent directement des fluctuations de la pousse de l'herbe. Par ailleurs, la production de fourrages à partir des prairies permanentes présente une très forte variation interannuelle due à la sécheresse et qui peut être amplifiée par des périodes froides de début de printemps. L'exploitant y perd davantage que dans un système de cultures : en 1976, la production du blé n'a été réduite que de 10 à $20 \%$ selon les régions, alors qu'en moyenne la production d'herbe a chuté de $50 \%$. La variation peut venir aussi de contraintes conjoncturelles. Entre 2000 et 2005, la surface consacrée aux fourrages diminue de $20 \%$ en Limousin, compensée, sans doute, par celle vouée au maïs grain : + $13 \%$. Les éleveurs doivent donc être vigilants et anticiper chaque année les effets de la conjoncture et ceux du climat en se basant non pas sur une production fourragère moyenne, mais sur un risque de sécheresse accepté, quitte à avoir un excès de fourrage en année humide ou normale. Contrairement aux systèmes de grandes cultures, les élevages d'herbivores ne peuvent pas s'adapter à une année " moyenne » en compensant les mauvaises années par les bonnes. 

sensibilité que ne laisserait pas soupçonner l'apparente abondance de la ressource en eau dans ce milieu de moyenne montagne océanique. D'où l'absolue nécessité de retenir l'eau lors d'épisodes pluviométriques d'hiver et de printemps, pour l'utiliser lors d'étiages. C'est l'irrigation, avec l'amélioration de ses équipements, qui contribue à l'extension des surfaces en maïs grain. Mais cette culture s'est développée à partir d'un système de stockage de l'eau, en réservoirs artificiels, et non par le biais de pompages en nappes, faute de réserves souterraines, comme c'est le cas sur les périphéries sédimentaires du Massif central. Au cours des dernières années l'accroissement de la production (321 000 quintaux en 2006) s'accorde avec celui des surfaces en maïs, dont plus des trois-quarts en Haute-Vienne et en Corrèze, pour un rendement moyen de 82 q/ha. 1100 hectares en 1981 ; en 1986 , elle passait à 1650 hectares, soit une part très faible de la SAU régionale (855 500 ha). De fait, il s'agissait presque exclusivement des parcelles de vergers, de petits fruits et de maïs, alors que l'irrigation des prairies perdait de son intérêt. Le coût des installations modernes d'irrigation et le petit nombre de réservoirs pour stocker l'eau ne pouvaient, à l'époque, justifier que l'on développe ces pratiques pour les herbages, alors que la valeur ajoutée des productions fruitières (pommes, petits fruits rouges) et légumières semblait un gage de rentabilité, avec un revenu plus élevé à l'hectare. C'est surtout le département de la Corrèze, le plus méridional du Limousin, qui tira profit de ce mouvement, avec ses traditions tabacole, fruitière et légumière. Dans les années 1980, l'irrigation en Limousin concerne plutôt les productions végétales, plus rentables.

24 Le système fourrager mis en place par l'éleveur intègre donc la perception qu'il a du risque de sécheresse, en fonction de la connaissance historique qu'il a acquise dans son exploitation. On parle alors de sécheresse anticipée pour définir les choix de l'exploitant face au risque sécheresse. Mais il peut s'agir aussi de sécheresse imprévue qui nécessite la mise en œuvre d'autres mécanismes de régulation du système d'élevage faisant le plus souvent intervenir des ressources fourragères, et d'autres moyens pour améliorer la productivité fourragère, par une maitrise de l'eau bien conduite et une gestion adaptée aux situations localisées : irrigation mais aussi drainage préalable des sols.

\section{Assainir les sols : le drainage raisonné « Transformer l'enfer en paradis »}

Le principe de base du drainage consiste à évacuer l'excès d'eau du sol - captage des sources, et des mouillères dans les fonds - pour favoriser une aération en profondeur et éviter l'asphyxie des plantes. Appliqué aux secteurs humides des fonds de vallées ou des milieux tourbeux, en tête de bassin - comme sur la Montagne limousine - il peut entraîner une modification $\mathrm{du}$ régime hydrique, mais son intérêt majeur reste l'optimisation des sols pour rentabiliser les productions agricoles dans des zones de faibles rendements. 
Photo 3 : «Limousines » dans un pré de fond (Plateau de Millevaches) (au second plan : parcelle en partie drainée [cl. F. Ardillier-Carras])

"Limousins " cattle in a wet meadow (Plateau de Millevaches) (At the background: plot partly drained)

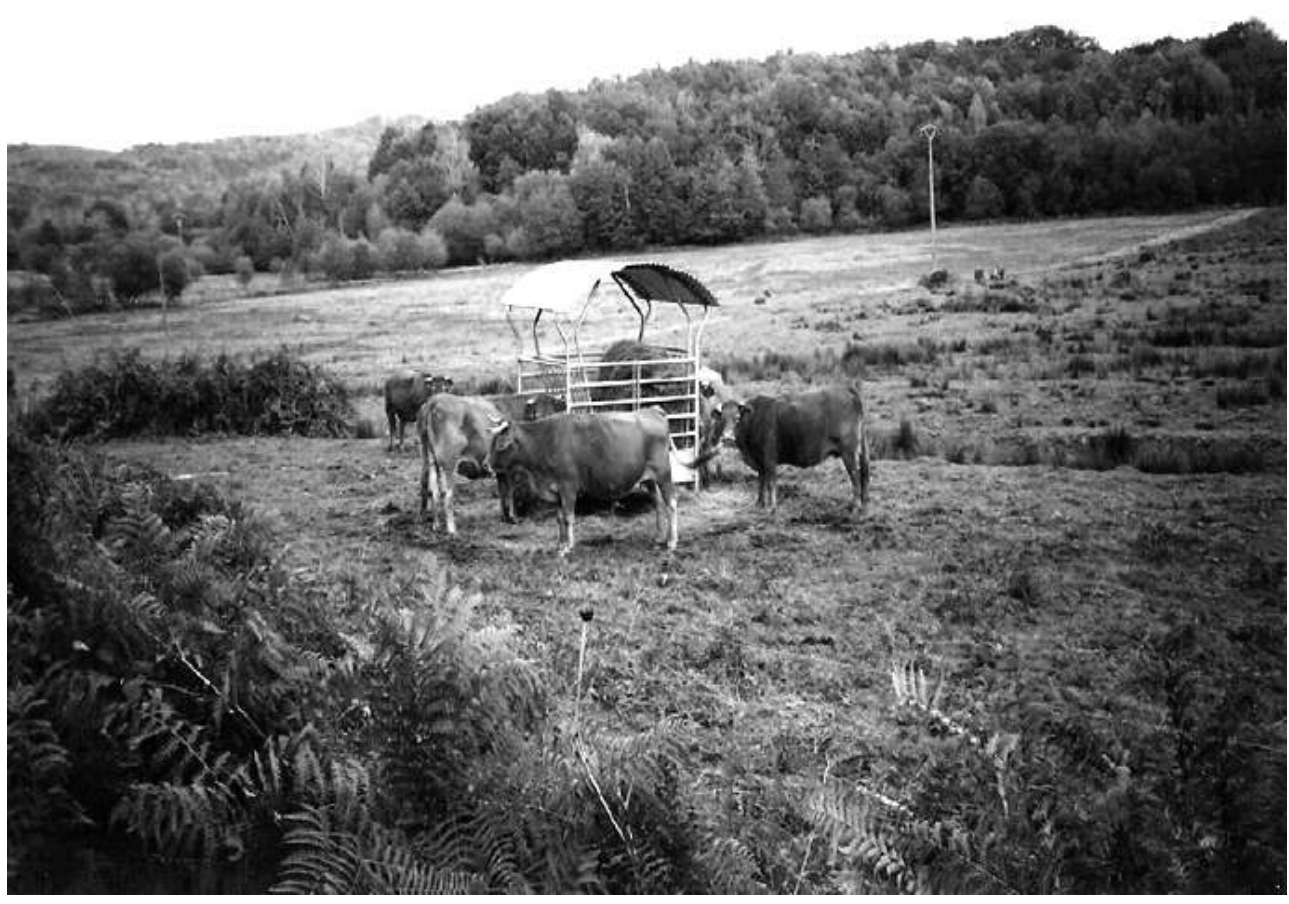

Le Schéma Régional d'hydraulique agricole lancé en 1979, pour le Limousin, évaluait à 200000 ha la superficie à drainer ou à assainir ${ }^{5}$. Il a été décisif pour le développement de certaines cultures liées à l'élevage. Des " associations syndicales autorisées de drainage " (ASAD) se constituèrent pour réaliser des opérations concertées sur de vastes surfaces. Mais des actions ponctuelles, à la parcelle, furent également menées par des agriculteurs pour traiter les zones de mouillères notamment. Les aides de l'État et de la Région ont permis un gros effort en faveur du drainage pour encourager des gains de productivité. L'objectif portait alors sur un ensemble de 1200 ha/an. Avec la sécheresse estivale des années 1985-1986 et 1987, cette initiative fut révélatrice de la nécessité de prendre en compte les épisodes climatiques excessifs : à la sécheresse peut succéder une période de forte humidité qui devient alors un facteur aggravant.

Transformer ainsi « l'enfer en paradis ${ }^{6}$ » va changer la vie des agriculteurs et vaincre les blocages techniques et sociaux du milieu paysan en Limousin. Au-delà de la capacité à faire évoluer les pratiques de maîtrise de l'eau, le vrai résultat de ces opérations a été cette dynamique de conquête des terres agricoles dans le but de les valoriser pour mieux produire et enclencher une dynamique vis-à-vis des agriculteurs limousins. Nombre d'exemples de drainage individuel se sont soldés par des échecs en raison des surcoûts et des investissements engagés ainsi que des aléas des revenus issus de la production de l'exploitation. Ce fut, par exemple, la triste expérience tentée par un couple de migrants installés en Haute-Vienne dans les années 1980. Vouée à l'élevage ovin viande, l'exploitation eut du mal à assurer un revenu suffisant et à rembourser les emprunts pour le drainage progressif sur ses 50 ha. Avec des annuités de l'ordre de 13 $000 €(85000 \mathrm{~F}$ de l'époque) et des revenus entamés par la baisse du cours de l'agneau, l'endettement s'installe et le processus d'amélioration des sols par drainage entraîne la faillite. Une mauvaise conjoncture au moment où le coût des emprunts pèse le plus lourd a eu raison de cette initiative. Quand irrigation et drainage sont étroitement 
associés, dans des exploitations plus viables, le résultat vient couronner de succès une logique innovante basée sur l'élevage bovin viande. Dans ce cadre, fortement intégré aux exigences de productivité, les pratiques de maîtrise de l'eau relèvent davantage encore d'une gestion raisonnée de la ressource, au plus près des réalités climatiques saisonnières, pédologiques, hydrologiques.

Figure 2 : Le drainage dans le bassin-versant de la Vienne. Cette carte du bassin-versant de la Vienne montre bien le contraste existant entre la moyenne montagne limousine (Haute-Vienne et Creuse) et les régions situées en aval où la culture du maïs a pu se développer sur les sols hydromorphes grâce à l'extension du drainage (Charente limousine et Vienne), alors que le Limousin reste avant tout un ensemble herbager, moins touché par cette technique.

The drainage in the catchment basin of the river Vienne

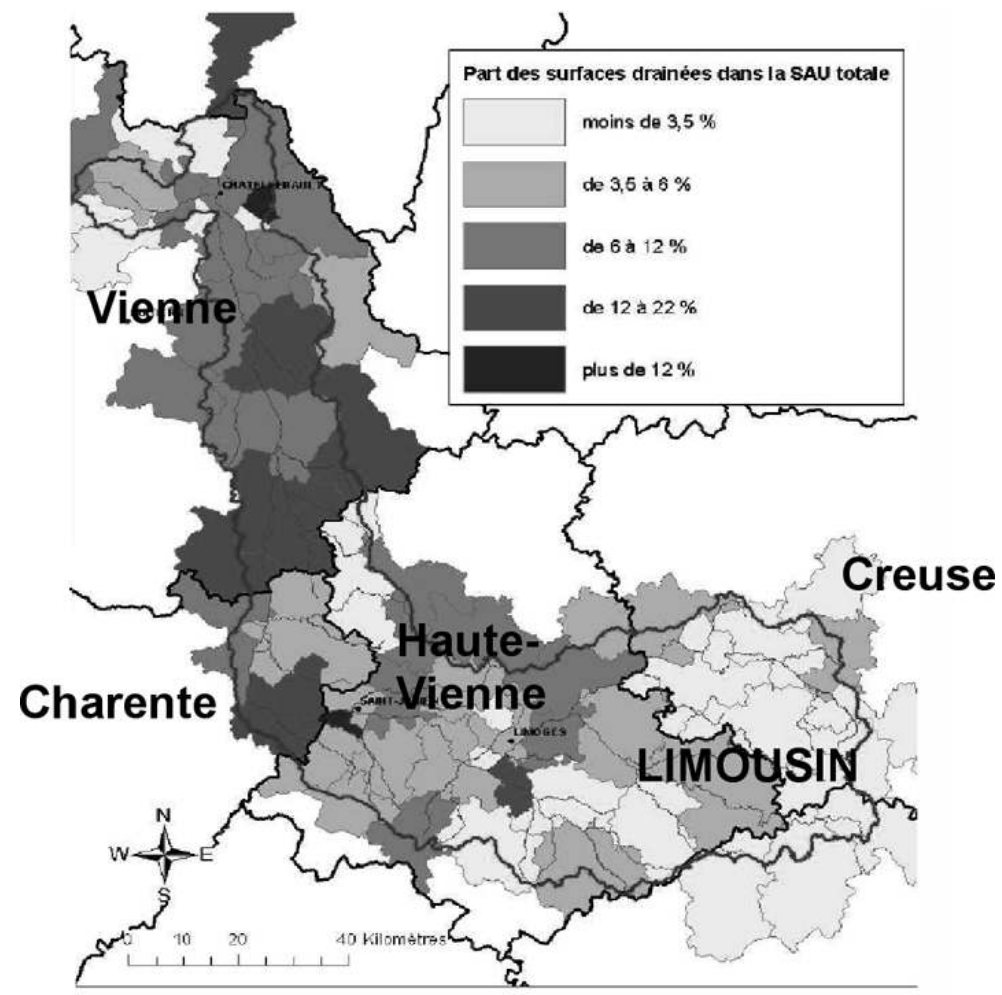

Le domaine de Berneuil, près de Bellac, est l'illustration des mutations radicales opérées grâce à la gestion de l'eau pour une production bovine d'envergure, associée à la production de maïs. Acquise en 1988 par un groupe franco-italien, cette exploitation de plus de 750 ha, vouée auparavant au mouton de plein-air, était pénalisée par des sols dégradés et une faible productivité. Drainage et irrigation ont été, d'emblée, étroitement associés. Le principe est conforme aux pratiques traditionnelles de maitrise de l'eau dans ce type de région : assainir les sols, drainer, récupérer les eaux en excès, stocker, irriguer uniquement à partir des réservoirs. Les 3 stockages, de 510000 $\mathrm{m}^{3}$, alimentés par les écoulements de surface, les eaux de pluie et de drainage, ainsi que par un transfert de réservoir à réservoir, aval-amont par pompage, constituent une armature parfaite pour pallier les manques estivaux et réguler les excès saisonniers. Avec des rendements en maïs de l'ordre de $150 \mathrm{q} / \mathrm{ha}$, sur 350 ha, cette entreprise agricole est autosuffisante pour l'alimentation du bétail (maïs, fourrages, blé, orge) et peut vendre les surplus de production.

Pari risqué, certes, et investissements importants pour un tel résultat : cette entreprise agricole « intégrée » s'appuie sur un terroir herbager et sa race à viande, la « Limousine 
", une association drainage-irrigation valorisant la ressource en eau, la complémentarité d'espaces au niveau européen entre le Limousin pour la production de «broutards ${ }^{7}$ » de race limousine et l'Italie, dans la plaine du Pô, pour l'embouche. C'est une gestion de l'eau, bien conduite, alliée à la fertirrigation ${ }^{8}$, qui a permis ce résultat, avec des capacités financières que n'ont pas les autres éleveurs de la région. Les contraintes du milieu naisseur-engraisseur ont été bien intégrées à ce schéma agissant comme une courroie d'entraînement. L'image ainsi véhiculée qu'il n'y a pas de fatalité, a bénéficié à des exploitants, malgré les risques inhérents aux aléas d'une telle relation eau-agriculture. Parler de l'inertie des uns contre l'innovation des autres ne résume pas la situation. Il serait trop simpliste de réduire le processus aux seules considérations comportementales. Quant au risque, il a un coût, celui de l'avenir de l'exploitation. Hormis la mise en valeur de terres par une hydraulique agricole bien maîtrisée, il s'agit surtout de rentabilité pour l'avenir : les investissements réalisés pour réhabiliter les sols ont permis de valoriser fortement le patrimoine foncier : de $460 €$ (soit environ $3000 \mathrm{~F}$ ) dans les années 1970, l'hectare peut atteindre, une fois valorisé, 2 $000 €$ à $3500 €$ pour les terres labourables. Au-delà de ce volet de maîtrise de l'eau, de gestion des excès et de réhabilitation des sols hydromorphes, il faut voir ce que représente réellement la mise en œuvre de programmes de drainage, tant sur les comportements des agriculteurs que pour la valorisation de l'exploitation. Gérer l'utilisation de l'eau va, ainsi, bien au-delà de ses objectifs techniques et économiques liés à la production agricole. Cela constitue le socle d'un progrès agronomique et d'une nouvelle relation au milieu physique.

Figure 3 : Un schéma de gestion intégrée pour la maîtrise de l'eau et la production bovine de viande de qualité en Limousin

Integrated management for controlling water and cattle production for meat of the highest quality in the region of Limousin

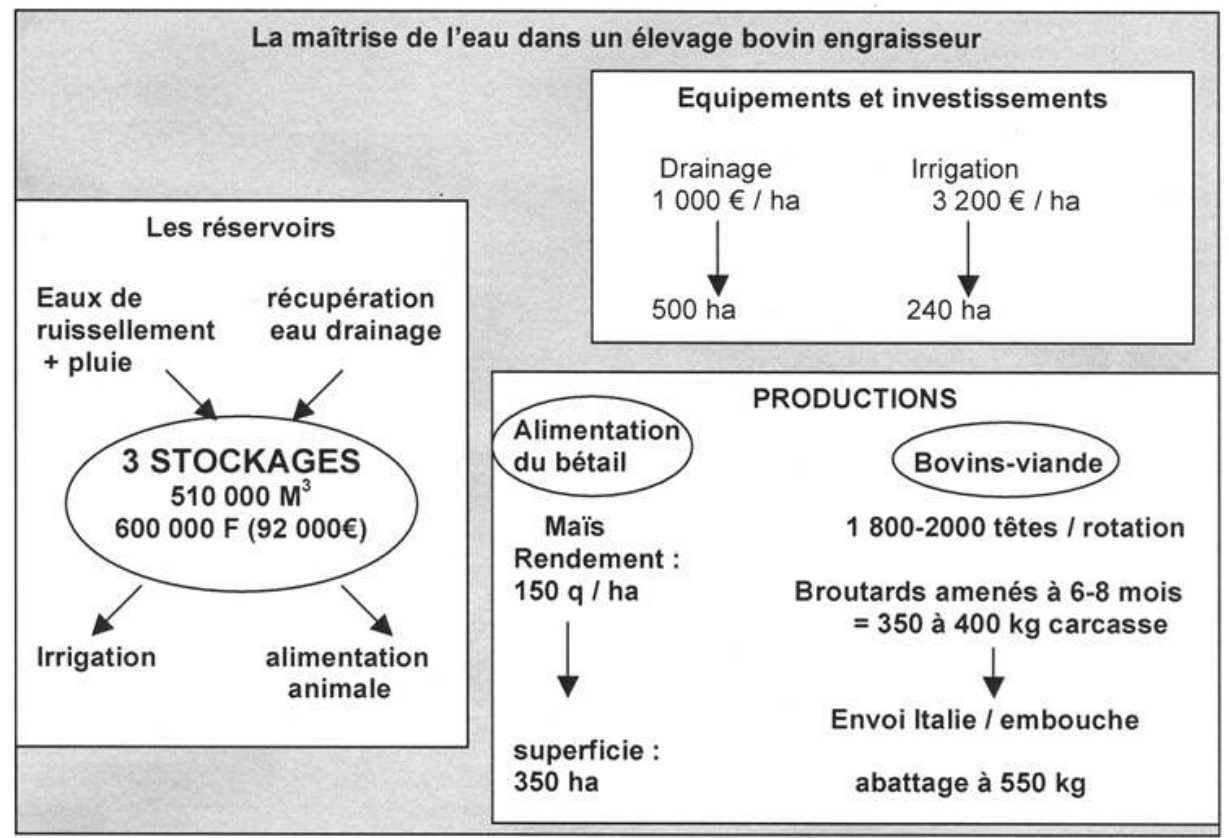

En définitive, il faut mettre en évidence les changements induits en termes d'aménagement et d'amélioration technique de l'exploitation, de restructuration du travail, voire des modes de production et de la structure du foncier comme du 
parcellaire. Le drainage a, en effet, été freiné par la taille et la forme des parcelles. Des opérations de regroupement parcellaire ou de remembrement ont dû être engagées pour rationaliser les opérations de drainage et d'irrigation. Hormis les caractères matériels de ces pratiques, l'exploitant est conduit à remettre en question la vocation et l'avenir de son exploitation. Dans une région où la majorité des élevages en bovinviande, n'ont pas de succession assurée, c'est le système tout entier d'une économie régionale de bassin allaitant qui se trouve confrontée à de sombres perspectives d'avenir. Que les subventions et aides européennes s'amenuisent, que les prix de vente de la production s'affaiblissent, qu'une crise du marché vienne balayer les revenus agricoles, et c'est la faillite pour l'exploitant qui a investi pour moderniser, en particulier dans de nouvelles techniques d'hydraulique. C'est ce que dénoncent les éleveurs du Limousin ${ }^{9}$ dans un journal professionnel titrant « l'élevage en danger », « le marché de la viande morose ", mettant en avant la détresse des exploitants de tout le bassin allaitant face à l'augmentation des charges. La diminution du nombre de broutards (« moins » 5000 têtes entre 2004 et 2005) en serait-elle une illustration ?

\section{Diversifier les productions en Limousin : des choix adaptés a la demande des marchés}

L'irrigation en Limousin n'est plus destinée prioritairement aux prairies, mais aux cultures capables de fournir un revenu élevé à l'hectare : vergers, petits fruits rouges, maïs pour l'alimentation animale. Le secteur des fruits rouges (framboises surtout, et fraises) s'est développé en Corrèze : 158 ha sur un total de 179 ha en Limousin. Mais surtout, depuis une vingtaine d'années la maîtrise de l'eau, couplée aux avantages de l'altitude et à la demande des marchés, a ouvert une autre voie : la production pommicole, couvrant environ 3000 ha, pour les $3 / 4$ en Corrèze, avec un total de 310 arboriculteurs. La " pomme du Limousin » est la seule pomme ayant obtenu une Appellation d'Origine Contrôlée (AOC), label français qui valorise aujourd'hui la Golden du Limousin. Il s'agit là d'une véritable démarche de valorisation du terroir par la recherche de la qualité et la mise en œuvre d'une gestion raisonnée de l'hydraulique agricole avecdensification du verger, limitation des risques (filets pare-grêle), irrigation goutte à goutte, raisonnement des pratiques culturales.

Produite entre 350 et $500 \mathrm{~m}$ d'altitude, pour la saveur du fruit, la pomme du Limousin a son label. Mais cette image de marque comporte un cahier des charges très strict où la notion de risque prend une réelle importance : non seulement en matière d'investissements et de débouchés, mais aussi en raison de la dépendance climatique (pluviométrie variable, grêle, gel tardif). En effet, notamment dans le nord du Limousin, l'implantation de vergers de pommiers n'est possible qu'avec l'amélioration des sols généralement hydromorphes : travaux de drainage couplés à un apport d'irrigation. Un temps de réponse de 5 à 6 ans est nécessaire avant le début de la rentabilisation. Avec 871000 quintaux par an (2005), le Limousin ne se positionne pas parmi les plus gros producteurs de pommes mais ceci démontre que le fait altitudinal de moyenne montagne, associé à l'utilisation maîtrisée de sa ressource en eau contribue à valoriser l'espace agricole autrement que par sa vocation herbagère. Un secteur agro-alimentaire actif relaie le producteur dans la commercialisation, avec ou sans transformation, d'un produit dont la collectivité territoriale (Conseil Régional) fait une intense promotion auprès des consommateurs, en France et à l'étranger. 
Photo 4 : Vergers de pommiers en Limousin : second producteur de "golden » en France (source : « Pomme labellisée AOC du Limousin »)

Apple orchards in the Limousin

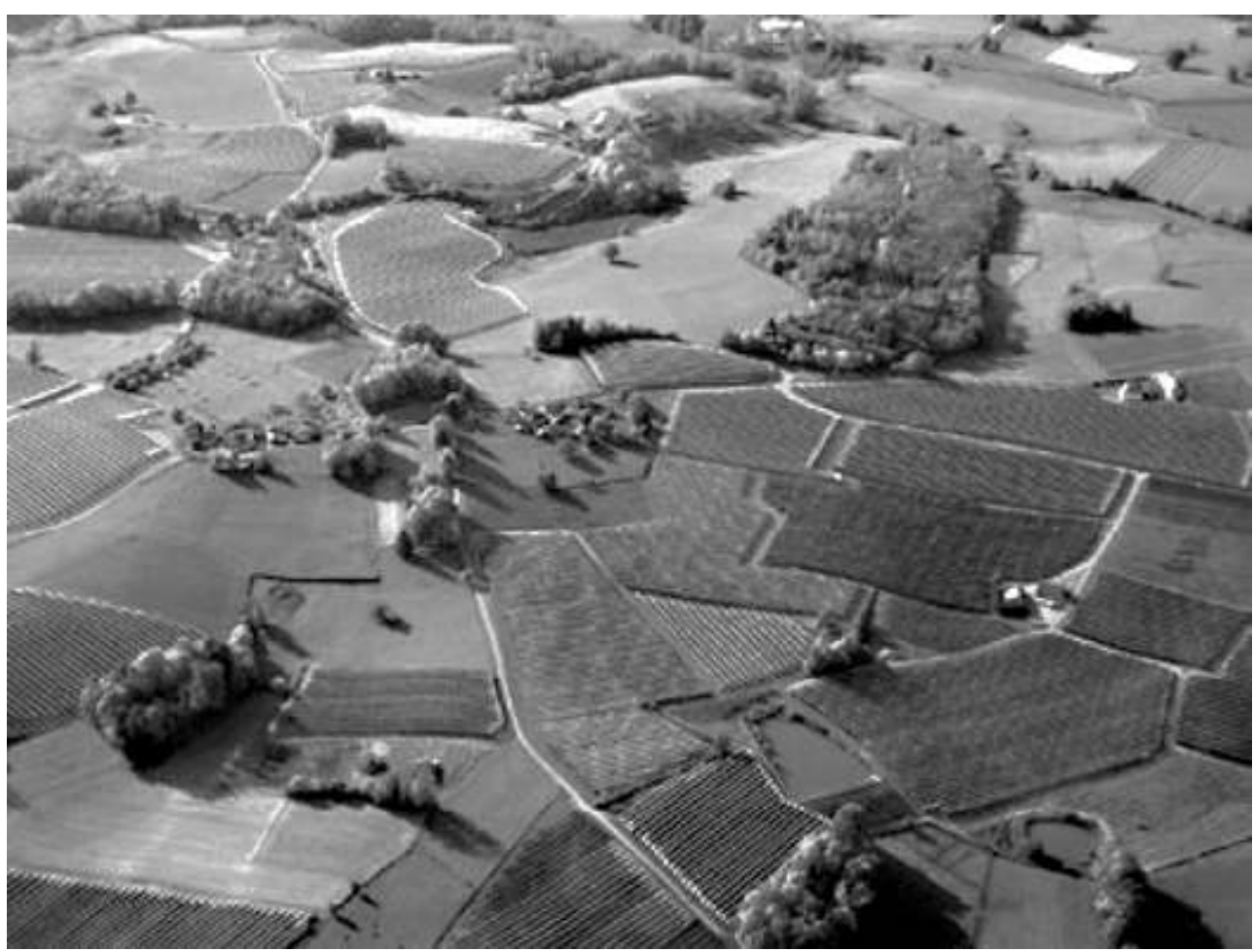

\section{Maîtrise de l'eau et agriculture de moyenne montagne dans le contexte de l'UE}

La conjoncture économique mondiale et la politique agricole de l'UE sont des leviers de changement, assortis d'incitations réglementaires, pour une meilleure gestion de la ressource en eau et l'adaptation des pratiques agricoles aux besoins environnementaux.Dans la nouvelle PAC, le versement des aides est conditionné au respect des directives en vigueur (maintien des zones humides, biodiversité...) et au respect de BCAE (Bonnes conditions agricoles et environnementales), définies par les États.

Après la réforme de la PAC de 1992, la France a choisi d'accorder une aide majorée aux cultures irriguées vis-à-vis des cultures en sec dans bon nombre de départements ; ceci a certainement contribué à la poursuite de l'accroissement de l'irrigation constaté entre 1994 et 2000. Si la logique des responsables européens est assez éloignée des préoccupations de certaines régions, notamment des zones herbagères, les risques d'atteinte à l'environnement sont une des préoccupations majeures, notamment par les impacts de l'hydraulique agricole sur la dégradation de la qualité de l'eau. Peut-on opposer, suivant les termes de Jacques Béthemont (1972), « un ordre traditionnel agricole et un ordre nouveau fondé sur la reconversion de l'hydraulique agricole »? 


\section{Les espaces de moyenne montagne : parents pauvres de l'agriculture européenne?} montre bien la faible part du Limousin, dont les apports viennent essentiellement des écoulements de surface. À l'inverse, la partie aval du bassin, sur les marges poitevines, sédimentaires, bénéficie largement des apports de l'amont auxquels s'ajoutent les réserves souterraines. Ainsi, l'eau issue de l'amont Limousin s'écoule sur son substrat granitique, vers un aval consommateur pour ses cultures irriguées, maïs en particulier. L'utilisation agricole de la ressource en eau, provenant du « château d'eau » limousin, se fait donc essentiellement au profit de l'aval, non producteur, mais qui possède de nombreux aquifères.

\section{Préserver la ressource et les écosystèmes dans les milieux vulnérables de têtes de bassins : les incitations européennes}

39 La protection des ressources en eau et leur gestion raisonnée sont deux volets essentiels de toute stratégie de développement durable et de maintien de la biodiversité. La préservation des milieux aquatiques est définie par de nombreux outils réglementaires nationaux et européens. Pour le Limousin, les orientations sont ancrées dans une logique de «tête de bassin ». L'agriculture y est peu polluante, basée sur des systèmes d'élevage en extensif (ou semi-extensif bovin), mais la vulnérabilité des milieux aquatiques, inhérente à la situation d'amont, justifie une vigilance toute particulière. Le petit chevelu hydrographique y reste très sensible aux impacts du drainage des fonds humides, au piétinement des animaux sur les rives ou à d'autres effets, même mineurs, liés à l'activité agricole.

- La loi sur l'eau ${ }^{10}$ en est le volet fondamental. Afin de réguler les usages de l'eau et de protéger la ressource, la loi de janvier 1992 introduit la notion de " patrimoine commun » et de gestion équilibrée au bénéfice de la protection des milieux naturels et instaure deux instruments de planification nouveaux : les SAGE et les SDAGE ${ }^{11}$. Le SDAGE Loire-Bretagne prend en compte les spécificités de l'amont des bassins versant. Pour la préservation des milieux dits « remarquables »- dont une partie concerne des 
milieux aquatiques - les SAGE définissent à l'échelle des bassins versants les termes de la gestion de l'eau et, tout particulièrement, l'hydraulique agricole. Les enjeux s'appliquent à la préservation de la ressource et des milieux, en partie par le contrôle des pratiques agricoles à l'amont, en Limousin. En sa qualité de " tête de bassin ", le Limousin est défini comme espace vulnérable : les zones humides du plateau de Millevaches doivent être prises en compte dans la politique des espaces naturels sensibles, au faible développement économique. Cette notion s'applique davantage à la qualité de l'eau et des milieux aquatiques qu'à la logique quantitative, d'où l'incitation formelle à des pratiques agricoles respectueuses des écosystèmes. On touche là une véritable ambiguïté : dans une région amont de bassin, bien alimentée en eau, la crainte de dégâts environnementaux, plus que le constat de dégâts réels, conditionne les préconisations réglementaires, dans un ensemble agricole d'extensif bovin viande au faible poids économique dans la politique agricole européenne.

41 - Le label Natura 2000 est une mesure résultant de directives européennes visant à la préservation des habitats et de certaines espèces d'oiseaux qui s'applique, entre autres, à des milieux humides, étangs, zones de sources. De ce fait, mal perçus par les agriculteurs, qui craignent de voir réduire leurs pratiques agricoles, les périmètres de Natura 2000 ont été fréquemment décriés par les acteurs de l'économie agricole.

- Les programmes agri-environnementaux, plus spécifiquement tournés vers l'agriculture et assortis d'incitations financières, confirment la nécessité impérative de protéger l'environnement. Le règlement (CEE) $n^{\circ}$ 2078/92 du Conseil, ou règlement agrienvironnemental, prévoit la mise en œuvre de programmes afin d'encourager les agriculteurs à développer des activités ayant des effets positifs sur l'environnement. Ces mesures prévoient la compensation financière des coûts auxquels ils doivent faire face et des pertes de revenus issus des moyens engagés pour respecter l'environnement.

Parmi les objectifs, il s'agit de réduire les effets négatifs de l'agriculture sur l'environnement, en particulier sur la qualité de l'eau, le sol et la biodiversité ainsi que de promouvoir les pratiques agricoles nécessaires au maintien de la biodiversité et du paysage. Quant aux contrats d'agriculture durable (CAD), ils privilégient la protection de l'eau et des milieux aquatiques et conditionnent ainsi les aides aux agriculteurs. La réglementation en vigueur dans l'UE (mesures agri-environnementales, directive nitrates, SAGE, contrats de rivières...) compose ainsi un ensemble de mesures de " gouvernance » de l'eau pour chacun des secteurs d'activités.

\section{Des choix commandés par les politiques environnementales}

En Limousin, comme dans d'autres régions de même type, la maîtrise de l'eau et des sols représente une victoire, cher payée, une revanche sur les contraintes du milieu. Elle rend possible la rentabilisation d'espaces défavorisés et ralentit les effets de la déprise agricole : le maintien en bon état du capital foncier reste un gage de survie dans un environnement économique fragile. En revanche, le refus de l'hydraulique ne révèle pas toujours une forme d'inertie. Il peut être à l'origine d'autres schémas, basés sur l'extensif raisonné. Cela peut procéder d'une démarche négative et conduire à l'abandon d'espaces agricoles, mais peut aussi relever d'une option réfléchie d'entretien du milieu et de préservation de la ressource, sans recours à l'hydraulique. Le « seuil » de rentabilité d'une production, comme le maïs, passe aussi par un plus 
faible rendement et des investissements (donc des charges d'emprunt) réduits. C'est le choix fait par des exploitants, parmi les plus jeunes, qui ont délibérément opté pour une moindre pression sur l'eau.

Pourtant, l'Europe subventionne les équipements hydrauliques, ce qui favorise leur installation, donc l'accroissement des prélèvements en eau. D'autre part, au nom de la préservation de la ressource (quantité et qualité), les organismes de gestion de l'eau, tels qu'Agences de bassins et organismes européens, vont pénaliser ces mêmes agriculteurs dès lors que des impacts environnementaux auront été relevés, consécutifs à l'utilisation de l'eau pour les cultures. Pour mieux mesurer les effets d'une réduction de l'irrigation, voici présentés dans le tableau ci-dessous les rendements du maïs selon les pratiques adoptées (moyenne en France).

\begin{tabular}{|c|c|c|c|c|}
\hline & \multicolumn{2}{|c|}{ Maïs non irrigué } & \multicolumn{2}{c|}{ Maïs irrigué } \\
\hline & Surfaces (ha) & Rendement $(\mathrm{q} / \mathrm{ha})$ & Surfaces (ha) & Rendement (q/ha) \\
\hline 2002 & 1027095 & 84 & 753055 & 101 \\
\hline 2003 & 900610 & $59(-30 \%)$ & 735160 & $89(-12 \%)$ \\
\hline 2004 & 1011183 & 83 & 755392 & 103 \\
\hline 2005 & 931476 & 74 & 683317 & 98 \\
\hline
\end{tabular}

Cette région agricole, qui sait depuis très longtemps développer de bonnes pratiques de gestion de l'eau, doit dans la conjoncture actuelle valoriser son savoir faire selon les exigences environnementales et économiques.

\section{Conclusion}

Il faut retenir de cet éclairage sur la petite hydraulique agricole dans une région de moyenne montagne sous influence océanique que la variabilité pluviométrique n'est plus seule en jeu. Aux contraintes physiques du milieu, à la faiblesse de cette agriculture mal rémunérée, s'ajoute une autre variabilité, celle des marchés, national et européen, des productions agricoles animales. S'y ajoute l'incessant ballet des primes et subventions - remèdes ou incitations - qui déstabilise le contexte économique des régions de production animale dont la réactivité est beaucoup moins souple que celle des productions végétales telles que les grandes cultures céréalières.

" Innover pour ne pas mourir » comme l'exprimaient des éleveurs lors d'une enquête 12 portant sur plus de 800 chefs d'exploitation en Limousin, serait l'expression de cette vision d'un avenir "sombre ». Pour $22 \%$ d'entre eux, les politiques agricoles européennes, par le jeu des orientations de production et de l'attribution de subventions, accentuent la notion de dépendance de cette agriculture de moyenne montagne. Quelle part revient alors aux pratiques agricoles basées sur la maîtrise de l'eau? Quelles formes de gestion doit aujourd'hui adopter l'exploitant du Limousin confronté aux impératifs économiques d'une région fragile où la non reprise des exploitations, - avec son corollaire d'agrandissement - conduit à des choix « par défaut »? La petite hydraulique agricole entre véritablement dans un schéma de modernisation des exploitations en accord avec les exigences environnementales. Elle s'avère encore plus indispensable pour le maintien des supports de production herbagère, gage de qualité de l'élevage bovin viande et de la valorisation de la race limousine, et reste essentielle pour mener une diversification vers des productions fruitières labellisées. Mais c'est aussi dans un objectif de valorisation patrimoniale, à but économique, qu'elle s'inscrit pleinement dans l'avenir de l'agriculture de moyenne 
montagne : la plus-value ainsi apportée au foncier agricole - par le redressement des sols et les apports en eau d'irrigation - contribue à encourager un processus de reprise des exploitations, y compris par des exploitants européens tentés par le faible coût des terres et le potentiel foncier disponible, notamment, des Néerlandais et des Britanniques. Il ne faut pas s'illusionner pour autant. Cette forme d'agriculture, peu rentable aux yeux d'une conjoncture mondialisée, risque de devenir une « niche » de production de qualité, dont les consommateurs ont besoin, mais qui ne se rentabiliserait qu'au prix des départs et de la déprise de l'espace agricole.

\section{BIBLIOGRAPHIE}

ARDILLIER-CARRAS (F.), 1997. - L'eau, ressource pour le développement d'un espace rural. Exemple du bassin de la Gartempe, Limoges, PULIM, 590 p.

ARDILLIER-CARRAS (F.), BALABANIAN (O.), 1998. -L'agriculteur dans son territoire, Chambre régionale d'agriculture du Limousin.

BALABANiAN (O.), BOUET (G.), 1989. - L'eau et la maîtrise de l'eau en Limousin, Treignac, Les Monédières, $296 \mathrm{p}$.

BETHEMONT (J.), 1972. - Le thème de l'eau dans la vallée du Rhône, Saint-Étienne, Thèse d'État.

DELHOUME (J.P.), 2004. - « L'élevage bovin en Limousin au XVIII ${ }^{\mathrm{e}}$ siècle. Des bœufs gras pour Paris », Histoire et sociétés rurales, $\mathrm{n}^{\circ} 22$.

GHIOTTI (S.), 2007. - Les territoires de l'eau. Gestion et développement en France, Paris, CNRS, coll. « Espaces et milieux $", 248 \mathrm{p}$.

HONNEGER (A.) et RUF (Th.) (dir.), 2005. - « La gestion sociale de l'eau, concepts, méthodes et applications ", Montpellier, revue Territoires en mutation, $\mathrm{n}^{\circ} 12$.

HONNEGER (A.) et RUF (Th.) (dir.), 2000. - « Approches sociales de l'irrigation et de la gestion collective de l'eau. Démarches et expériences en France et dans le monde », Montpellier, revue Territoires en mutation, $\mathrm{n}^{\circ} 7$.

MIGNON (ch.), 1997. - « Le rôle de l'agriculture dans les moyennes montagnes françaises », dans Gestion des espaces fragiles en moyenne montagne. Massif central-Carpates polonaises, Clermont-Ferrand, CERAMAC, p. 11-26.

PERPILlOU (A.), 1950. - Le Limousin. Etude de géographie physique régionale, Chartres, Imp. Durand.

SIMON (A.), 2003. - «Crises et adaptation des exploitations agricoles : l'exemple de la moyenne montagne creusoise ", dans Crises et mutations des agricultures de montagne, Clermont-Ferrand, CERAMAC, p. 603-617.

TimbAL (L.), schmitt (A.), 1950. - La région limousine, Limoges, Ch. Lavauzelle, 176 p.

Ministère de l'Agriculture et de la Forêt, 2006, Statistiques régionales. 
PLANEL (S.), 2003. - Crises et mutations des agricultures de montagne, CERAMAC, Presses

universitaires de Clermont-Ferrand.

YoUNG (A.), 1931. - Voyages en France en 1787, 1788 et 1799, Paris, Armand Colin.

\section{NOTES}

1. Slogan figurant sur les documents touristiques du Limousin.

2. «Le pré de fauche est le fond le plus estimé, son revenu représentant six fois celui d'une terre à seigle » (Delhoume, 2004).

3. La « nourriture hivernale des bovins est constituée principalement de foin, lequel est produit dans un espace spécifique, le pré de fauche ", selon une surface proportionnée au nombre de bovins adultes (ibidem).

4. Cette irrigation à finalité thermique était aussi pratiquée dans la plupart des massifs hercyniens européens.

5. Soit 55000 ha, ou $25 \%$ des superficies à drainer, et 135000 ha, ou $20 \%$ de la STH (Surface Toujours en Herbe) à assainir.

6. Les migrants, à l'origine de cette technique en Limousin, expriment ainsi les changements vécus dans leurs exploitations.

7. Animaux maigres, de moins d'un an, après une saison de pâturage avec la mère.

8. Ici : adjonction de fertilisants d'origine animale (rejets d'élevage) à l'eau d'irrigation.

9. L'Union agricole, 25 avril 2008.

10. La loi $n^{\circ} 92-3$ du 3 janvier 1992 et la loi $n^{\circ} 2006-1772$ sur l'eau et les milieux aquatiques a été promulguée le 30 décembre 2006.

11. SDAGE : Schéma Directeur d'Aménagement et de Gestion des Eaux; SAGE : Schéma d'Aménagement et de Gestion des Eaux.

12. Enquête réalisée pour la Chambre d'Agriculture du Limousin en 1998 sur l'avenir des exploitations en Limousin (Ardillier-Carras et Balabanian).

\section{RÉSUMÉS}

Maîtriser l'eau pour l'agriculture est une préoccupation ancienne et contemporaine sous bien des latitudes. Les pratiques de gestion de la ressource et les techniques se sont modernisées dans un contexte de productivité et de concurrence des marchés. Le Limousin, région de moyenne montagne en bordure nord-ouest du Massif central, à vocation herbagère, est bien approvisionné en eau, mais subit malgré tout des manques et des excès auxquels les agriculteurs doivent faire face. L'évolution actuelle de son économie agricole tient compte des conditions du milieu, des impératifs du marché, des politiques européennes et de nécessités économiques et environnementales parfois contradictoires. Inscrite dans un plus vaste débat sur le rôle des agricultures de montagne, la question de la maîtrise de l'eau révèle aussi les faiblesses des régions de moyenne montagne partagées entre préservation de la ressource en eau, amélioration des rendements et fragilité économique. 
Controlling water for the purposes of agriculture has been, for centuries, a major preocupation throughout the world. Modern techniques have been developed in a content of competitive markets and productivity. In the Limousin, a region of medium mountains on the North-West border of the Massif central, there is extensive grazing land and water supplies are abundant. Nevertheless, farmers have to contend with either excessive or insufficient rainfall from one season to another. The current evolution of agricultural economies in the Limousin depends on a number of factors: the physical conditions of the environment, the demands of the market, European policy and economic and environmental requirements which may be in opposition to one another. Seen within the larger context of the role of mountain agriculture, the question of controlling water also reveals the inherent weakness of medium mountains regions, struggling between the need to preserve their water resources, improved production and economic fragility.

El dominio del agua para la agricultura es una preocupación antigua y contemporànea en diversas latitudes. Las practicas de gestión del recurso y las técnicas se han modernizadas en un contexto de productividad y de competencia de los mercados. En el Limousín, región de media montaña, en el borde Nord Oeste del Macizo Central francés, con vocación ganadera de pastizales, el agua abunda, pero los agricultores deben hacer frente a pesar de todo a las carencias y a los excesos según las estaciones y los años. La evolución actual de su economía agrícola esta condicionada por lo físico, los imperativos del mercado, las políticas de la U.E. y las necesidades económicas y medioambientales a veces contradictorias. Inscrita en un debate muy amplio respecto al papel de las agriculturas de montaña, la cuestión del dominio del agua revela también la debilidad de los espacios de media montaña divididos entre preservación de este preciado recurso, la mejora de los rendimientos y la vulnerabilidad económica.

\section{INDEX}

Palabras claves : diversificación agrícola, dominio del agua, ganadería de pastizales, media montaña oceánica, medio ambiente, producciones agrícolas, recursos hídricos, U.E.

Index géographique : Limousin, Massif central

Mots-clés : diversification agricole, environnement, gestion des ressources, ressources naturelles, production agricole, Union européenne

Keywords : agricultural diversification, agricultural production, environment, resource management, European Union, natural resources, breeding

\section{AUTEUR}

\section{FRANÇOISE ARDILLIER-CARRAS}

EA 1210 CEDETE - Orléans, Université de Limoges, 39E rue C.-Guérin - 87036 Limoges cedex, France, ardillier.carras@wanadoo.fr 